\title{
Test de figura humana en pacientes en edad pediátrica con antecedentes de abuso sexual. Estudio de casos
}

\author{
Rodríguez $\mathrm{J}^{\mathrm{I}}$, Minck $\mathrm{N}^{\mathrm{I}}$, Acosta $\mathrm{M}^{\mathrm{I}}$, Cristina Arrom ${ }^{\mathrm{I}}$, Romero $\mathrm{M}^{\mathrm{I}}$, Arrom $\mathrm{CM}^{\mathrm{II}}$ \\ I. Cátedra de Psiquiatría-Facultad de Ciencias Médicas de la Universidad Nacional de Asunción, Paraguay. \\ II Centro para el Desarrollo de la Investigación Científica CEDIC/Díaz Gill, Paraguay
}

Cómo referenciar este artículo/ How to reference this article:
Rodríguez J, Minck N, Acosta M, Arrom C, Romero M, Arrom CM. Test de figura humana de niñas con antecedentes de abuso sexual. Estudio de casos. Mem. Inst. Investig. Cienc. Salud. $2015 ; 13(2): 75-81$.

\section{RES U M E N}

Se planteó evaluar presencia de indicadores de abuso sexual en la infancia (ASI) con indicadores de Boscato en los test de figura humana de niñas con y sin antecedentes de ASI en la Cátedra de Psiquiatría del Hospital de Clínicas. Estudio exploratorio, de corte transversal. Se realizó un análisis de test gráficos de figura humana en 16 pacientes: 8 con antecedentes de ASI y 8 sin ASI. Intensidad de la agresión: Descarga motora: en 6 del total de 8 pacientes con ASI, contra sin hallazgo en pacientes sin ASI. Concepción de sí mismo: percepción de estar traumatizado, en 2 de los 8 pacientes con ASI y en ninguna sin ASI. Micrografía: no presente en las pacientes con ASI en cambio sí en 1 de las pacientes sin ASI. Negación: en 2 del total de pacientes con ASI y en ningún paciente sin ASI. Vulnerabilidad (necesidad de defensa): en 6 del total de pacientes con ASI y en ningún paciente sin ASI. Percepciones sensoriales: un signo presente en 1 paciente sin ASI y ausente en pacientes con ASI. Registro somático, Heridas o marcas en el cuerpo: en $6(75 \%)$ del total de pacientes con ASI y en 2 de pacientes sin ASI. Se hallaron indicadores específicos para detección del abuso sexual Infantil que se corresponden con Intensidad de la agresión, concepción de sí mismo y en menor grado: registro somático y relación social.

Palabras clave: test de figura humana-indicadores de abuso sexual infantilepoblación pediátrica.

\section{Test of human figure in children with history of sexual abuse. Case study}

\section{A B S T R A C T}

The objective was to evaluate the presence of sexual abuse indicators (SAI) in childhood using the Boscato human figure test in girls with and without a history of SAI in the Department of Psychiatry of the Hospital de Clinicas. It is a exploratory, cross sectional study. An analysis of the human figure graphics test was performed in 16 patients: 8 with a history of SAI and 8 without SAI. Intensity of aggression: Motor skills: 6 of the total of 8 patients with SAI against no findings in patients without SAI. Conception of himself: perception of being traumatized in 2 of 8 patients with SAI and none without SAI. Micrographia: not present in patients with SAI but present in 1 patient without SAI. Denial: 2 of all patients with SAI and none in patients without SAI. Vulnerability (need of defense): 6 of all patients with SAI and no patient without SAI. Sensorial perceptions: one sign present in 1 patient without SAI and absent in patients Fecha de recepción: febrero 2015. Fecha de aceptación: junio 2015.

Autor correspondiente: Marina Arrom. Centro para el Desarrollo de la Investigación Científica CEDIC/Díaz Gill, Paraguay

E-mail: marinaarrom@yahoo.com.ar 
with SAI. Somatic register; Wounds or marks on the body: in 6 of all patients with SAI and in 2 of patients without SAI. Specific indicators of child sexual abuse detection were found that correspond to the intensity of the aggression, the self-conception and to a lesser degree : to somatic register and social relationship.

Keywords: test of human figure-indicators of child sexual abuse- pediatric population.

\section{INTRODUCCIÓN}

En el año 2006, Naciones Unidas publicó un estudio internacional que pone en evidencia la dimensión del maltrato infantil y sus devastadoras consecuencias, señalando que 80.000 niños y niñas pierden la vida cada año en América Latina como consecuencia de la violencia doméstica. (1).

En la región la violencia contra los menores de edad al interior de las familias se manifiesta principalmente a través del castigo físico como una forma de disciplina, el abuso sexual, el abandono y la explotación económica. (1).

Actualmente en Paraguay aproximadamente el $40 \%$ de la población está constituida por niñas, niños y adolescentes (2). En los últimos años se pudo observar un aumento en las denuncias de violencia en contra de ellos según datos que se desprenden de un balance presentado en el mes de mayo por el servicio de Fonoayuda - línea 147 (emprendimiento llevado a cabo por la Secretaria Nacional de la Niñez y la adolescencia) (3).

Para la Unicef el Abuso Sexual: comprende los contactos e interacciones entre un niño y un adulto, cuando el adulto (agresor) usa al niño para estimularse sexualmente él mismo, al niño o a otra persona (4). Un estudio probabilístico representativo de estudiantes de Alto Paraná, Paraguay, mostró que 8,6 tenía antecedentes de abuso sexual (5). En una muestra de 163 pacientes de 3 a 18 años de la Cátedra de Psiquiatría se encontró que 9,2 había padecido abuso sexual solo o combinado con otras formas de violencia intrafamiliar (6).

En un estudio exploratorio sobre maltrato escolar en Paraguay, Base/Plan/MEC (2014) (7), que incluyó ocho departamentos y establecimientos educativos de gestión pública oficial, los casos agrupados como violencia sexual configuraron el 42 por ciento de los 122 registrados (8). Las denuncias por abusos sexuales en niños, niñas y adolescentes en el país pasaron de 788 en 2005 a 2.298 en 2010, según un comunicado del Fondo de la ONU para la Infancia (Unicef) (9).

La experiencia de abuso sexual en la infancia constituye un importante factor de riesgo para el desarrollo de una gran diversidad de trastornos psicopatológicos en la edad adulta (10).

Putnam en su revisión de los estudios publicados sobre consecuencias psicológicas del abuso sexual infantil, destaca la gran variedad de condiciones psiquiátricas que se han asociado de forma consistente con la experiencia de abuso, como los trastornos depresivos, el trastorno límite de la personalidad, el trastorno de somatización, los trastornos relacionados con substancias, el trastorno por estrés postraumático, los trastornos disociativos y la bulimia nerviosa (10). Lo que hace que sea más complejo del abuso sexual es que se da justamente en los lugares en donde debieran ser espacios de protección y cariño. Debido a la falta de autonomía de los niños y las niñas estos son especialmente vulnerables al maltrato ya que no poseen el poder para protegerse o pedir ayuda.

Como el diagnóstico no es fácil suele pasar desapercibido durante mucho tiempo. La confirmación diagnóstica también es difícil y se basa en el relato del niño, sus juegos, su conducta, los dibujos, la historia clínica, el examen físico y los exámenes complementarios. Los signos físicos de certeza como los relacionados con enfermedades venéreas, desgarros en zona genital o embarazo son encontrados solo en la minoría de los casos (11). Muchos niños no lo manifiestan de forma espontánea o 
verbal en las consultas pero muchas veces aquello que no puede ser puesto en palabras se expresa a través del dibujo, juego, actos violentos, pesadillas etc. (12).

Históricamente, el hombre, ha utilizado dibujos como forma de registrar sus sentimientos y acciones; aun antes que símbolos que registraran el habla. La comunicación pictórica constituye un lenguaje básico y elemental siendo la primera forma de expresión que utiliza el niño y permite trabajar con niños pequeños sobre aspectos traumáticos, sin producir revictimización. (12).

Dentro de las pruebas gráficas se encuentra el Dibujo de la Figura Humana (DFH), creada por Karen Machover en 1949. La base teórica que sustenta esta prueba señala que un individuo frente a la instrucción: "Dibuje una persona", se halla obligado a dibujar partiendo de algunos referentes de apoyo. Las figuras externas son muy variadas en sus características corporales para presentarse espontáneamente en un dibujo, por lo que la persona debe realizar un proceso de selección de ciertos atributos. El individuo debe dibujar, consciente e inconscientemente, sobre su sistema completo de valores psíquicos, "siendo el cuerpo, o el propio yo, el punto más íntimo de referencia de cualquier actividad". (13).

Mediante esta prueba el sujeto vehiculiza aspectos de la personalidad en relación a su autoconcepto e imagen corporal, comprendiendo que "el dibujo de una persona, al envolver la proyección de la imagen de un cuerpo, ofrece un medio natural de expresión de las necesidades y conflictos del cuerpo de uno". (13).

En este trabajo nos centraremos en la expresión grafico plástica de dos grupos de Niños y Niñas del Servicio de la Cátedra de Psiquiatría con AS y sin ASI para comprobar los hallazgos de Boscato et al, aunque en una muestra pequeña por conveniencia y su importancia como herramienta para la sospecha de este antecedente en la consulta clínica para mejorar la detección precoz y la intervención para un mejor manejo de los casos. SE propuso entonces evaluar la presencia de indicadores de abuso sexual utilizando la investigación de Boscato en los test de figura humana de niños con antecedentes de abuso sexual y sin antecedentes de ASI en la Cátedra de Psiquiatría del Hospital de Clínicas.

\section{PACIENTES Y MÉTODO}

Es un estudio exploratorio, de corte transversal. Se realizó un análisis de test gráficos de figura humana en 16 pacientes: 8 con antecedentes de abuso sexual y 8 sin ASI, que acudieron a consultas psicológicas en el Hospital de Clínicas y contaban con Test de Figura humana; La selección de los test de pacientes se efectuó tomando los casos de una base de 163 pacientes del Servicio, cuyas edades oscilaban entre 3 a 18 años. Iniciando en forma consecutiva desde la primera ficha en adelante, se buscaron 8 casos de pacientes que habían sido víctimas de ASI y otros 8 del mismo sexo (4 varones y dos mujeres en cada grupo) y rango de edad que no habían sido víctimas de ASI.

Como se propuso evaluar la presencia de indicadores de abuso utilizando los hallazgos de Boscato y cols, en los test de figura humana de pacientes del Servicio como instrumento, se utilizaron 5 de los 7 indicadores de ASI de los autores, con los signos que los hacían observables a partir de sus investigaciones. (12). Dos de los indicadores, Expresiones de tipo sexual y Relación con el agresor no fueron considerados por no haberse registrado los datos al momento de la aplicación de los Test. Fueron considerados los indicadores de Intensidad de la egresión donde se observa el "grado de virulencia de la agresión sufrida y la capacidad subjetiva del niño para soportar el ataque", con los signos observables de personajes con armas (de fuego, cuchillos, flechas o elementos contundentes), descarga motora, escenas que sugieren situación de ataque; Concepción de sí mismo que "detecta la imagen psíquica que el niños posee de sí mismo, cuyos signos representan angustia, vivencias 
traumáticas, fortaleza, superioridad de condiciones o indefensión", a través de con sus signos, percepción de estar traumatizado, micrografía, negación, regresión, transparencias, vulnerabilidad (necesidad de defensa); Percepciones sensoriales donde el niño "da cuenta de percepciones que solo pudieron ser producidas por la propia experiencia a través de alguno de los sentidos", sus signos son pinches, vello; suciedad y manchas; elementos pegajosos y persistencia de representación de objetos de forma física con gotas o chorros; Registro somático, "representación gráfica de los efectos directos del trauma padecido sobre el esquema corporal" con heridas o marcas en el cuerpo, alteración u omisión de alguna zona corporal; Y por último Relación social "cuenta la actitud que asume el niño en el intercambio con el otro (aislamiento, negativismo, etc.)" con signos de aislamiento, secreto, hipervigilancia y necesidad de defensa. (12) Los datos fueron procesados utilizando Microsoft Office Excel 2010 para Windows.

El anonimato y la confidencialidad de la información del estudio se resguardaron utilizando un código numérico para cada Test.

\section{RESULTADOS}

Fueron incluidos en el estudio 16 pacientes, 8 con abuso sexual en la infancia (ASI) y 8 sin ASI. Los pacientes con ASI tenían 29 indicadores contra 16 en pacientes sin ASI.

Intensidad de la agresión: El signo descarga motora se encontró en 6 del total de 8 pacientes con ASI, en comparación con ninguno de los pacientes sin ASI. Puede interpretarse tanto como resultante de la sobreexcitación sexual, como de la dificultad vivenciada por el niño para "salirse" de la situación en que se siente atrapado. Se observa en el sombreado excesivo o sobrepasado de los límites del sector a colorear. Estas manifestaciones por lo general son acompañadas por actitudes y gestos que denotan ansiedad, angustia, ensimismamiento y en algunos casos desesperación. (Tabla 1)

Concepción de sí mismo: En este indicador se encontraron los siguientes signos: percepción de estar traumatizado, en 2 de los 8 pacientes con ASI y en ningún paciente sin ASI; el registro de vivencias traumáticas puede encontrarse plasmado en el gráfico mediante extremidades seccionadas, cicatrices o heridas y agujeros en partes del cuerpo a través de signos que representan angustia, vivencias traumáticas, fortaleza, superioridad de condiciones o indefensión. Micrografía: la micrografía no se encontró en las pacientes con ASI en cambio sí en 1 de los pacientes sin ASI. Este signo se infiere por el tamaño pequeño de la producción del niño y muestra la percepción que posee de sí mismo con relación al mundo externo. Negación: este signo se encontró en 2 de los 8 pacientes con ASI y en ningún paciente sin ASI. La implementación de la negación como mecanismo defensivo, muestra el intento del niño de "no querer ver" algún aspecto que lo perturba. Vulnerabilidad (necesidad de defensa) se encontró en 6 de los 8 pacientes con ASI y en ningún paciente sin ASI. Puede estar representada por personajes muy pequeños, extremadamente débiles o que experimentan una amenaza desproporcionada. (Tabla 1 )

Percepciones sensoriales: Sólo se encontró un signo (suciedad y manchas) de percepciones sensoriales en 1 paciente sin ASI y en ningún paciente con ASI. (Tabla 1)

Registro somático. Respecto a este indicador que consiste en la representación gráfica de los efectos directos del trauma padecido, sobre el esquema corporal, se encontró: Heridas o marcas en el cuerpo: en 6 de los 8 pacientes con ASI y en 2 pacientes sin ASI. Representado por cicatrices o marcas varias (magullones, 
sombreado sectorizado, etc.). La Alteración u omisión de alguna zona corporal: que evidencia conflicto a nivel corporal físico o de funcionamiento se encontró en 5 de los 8 pacientes sin ASI y en 3 de los 8 pacientes con ASI. (Tabla 1)

Relación social: En indicador que da cuenta de la actitud que asume el niño en el intercambio con el otro. En pacientes con ASI, se encontró el Signo del Secreto: ausencia de boca, boca enrejada, bocatapón, y otros detalles que revelan la "prohibición" de contar. También es posible hallar este signo en las manos escondidas tras la espalda o en bolsillos, mirada oculta por gorro de las figuras humanas, etc. Deja al descubierto lo que el niño guarda en secreto, generalmente por mandato del ofensor o por pudor. Y por último, en el grupo sin ASI se encontró la presencia de Hipervigilancia en 4 de los 8 pacientes sin ASI y en 2 de pacientes con ASI, que es el estado de alerta excesivo en el que se halla el niño. (Tabla 1)

Tabla 1. Indicadores de abuso sexual en pacientes con y sin ASI

\begin{tabular}{|c|c|c|c|}
\hline $\begin{array}{l}\text { Indicadores de abuso } \\
\text { sexual }\end{array}$ & Signos & $\begin{array}{l}\text { Con ASI } \\
\mathbf{n}\end{array}$ & $\begin{array}{l}\text { Sin ASI } \\
\mathbf{n}\end{array}$ \\
\hline \multirow[t]{3}{*}{ Intensidad de la agresión } & $\begin{array}{l}\text {-Personajes con armas (de fuego, } \\
\text { cuchillos, flechas o elementos } \\
\text { contundentes }\end{array}$ & - & - \\
\hline & -Descarga motora & 6 & - \\
\hline & $\begin{array}{l}\text {-Escenas que sugieren situación de } \\
\text { ataque }\end{array}$ & - & 1 \\
\hline \multirow[t]{6}{*}{ Concepción de sí mismo } & -Percepción de estar traumatizado & 2 & - \\
\hline & -Micrografía & - & 1 \\
\hline & -Negación & 2 & - \\
\hline & -Regresión & - & - \\
\hline & -Transparencias & - & 1 \\
\hline & -Vulnerabilidad (necesidad de defensa) & 5 & - \\
\hline \multirow[t]{3}{*}{ Percepciones sensoriales } & -Pinches, vello & - & - \\
\hline & -Suciedad y manchas & - & 2 \\
\hline & $\begin{array}{l}\text {-Elementos pegajosos y persistencia de } \\
\text { objetos de forma fálica con gotas o } \\
\text { chorros }\end{array}$ & - & - \\
\hline \multirow[t]{2}{*}{ Registro somático } & -Heridas o marcas en el cuerpo & 6 & 2 \\
\hline & $\begin{array}{l}\text {-Alteración u omisión de alguna zona } \\
\text { corporal }\end{array}$ & 3 & 5 \\
\hline \multirow[t]{4}{*}{ Relación social } & -Asimetría entre los personajes & - & - \\
\hline & -Secreto & 3 & - \\
\hline & -Hipervigilancia & 2 & 4 \\
\hline & -Necesidad de defensa & - & - \\
\hline
\end{tabular}




\section{DISCUSIÓN}

Casi el doble de cantidad de indicadores de abuso fue encontrado en el Grupo con ASI, en relación al grupo sin ASI. La diferencia es llamativa, dado el número muy bajo de casos analizados en el grupo de estudio. Aunque no se han consignado dos indicadores de Boscato. (12) El indicador de intensidad de la agresión encontrado se vincula estrechamente con el de relación con el agresor (que no fue utilizado en el presente estudio) y, según las investigaciones precedentes (12) revelan el grado de virulencia de la agresión y la capacidad subjetiva del niño para soportar el ataque, y el de Concepción de sí mismo encontrado en mayor proporción también evidencia la presencia de angustia y vivencias traumáticas e indefensión. (12). Es de destacar que estudios precedentes ya han confirmado la gravedad de los problemas de salud mental en otras etapas de desarrollo que pueden persistir en personas que han sido víctimas de abuso sexual en la infancia, como los estudios de Rodríguez López (13), donde se resalta la necesidad de detectar la problemática para realizar una intervención. (13).

Entre los indicadores específicos para detección del Abuso sexual Infantil encontrados en el grupo con ASI, aquellos de mayor puntuación se correspondieron con Intensidad de la agresión y Concepción de sí mismo, y en menor grado con Registro somático y Relación social. Para contar con mayor fuerza en los resultados del presente estudio de casos, se requiere ampliar el tamaño de la muestra y la cantidad de Test Gráficos aplicados, así como realizar el registro de la conducta de la población en consulta para evaluar la totalidad de los indicadores planteados por Boscato. Los resultados refuerzan hallazgos de los autores citados respecto a indicadores de abuso sexual infantil en Gráficos y requieren de mayor intensificación como aporte para el peritaje forense.

Si tomamos en cuenta las consecuencias en la vida futura de la población de estudio afectada por ASI, según Boscato, Ortelli, Sobrero (12), ellas estarían sujetas a la interacción de tres factores que se señalan, la estructura psíquica de la víctima, es decir los recursos emotivos, intelectuales y físicos con que cuenta ese niños, niña o adolescente para elaborar la agresión, la capacidad de contención del ámbito familiar para poder enfrentar la situación brindando protección y el tratamiento que el sistema judicial dé a ese delito en cuanto a la celeridad, características del proceso que se siga y sanción al agresor. (12)

Cabe señalar que el Dibujo de la Figura Humana se sostiene actualmente como un instrumento clave de batería diagnóstica y como parte fundamental de ella, resulta crucial a hora de evaluar daño emocional en niños, niñas y adolescentes.(14). Utilizando la expresión gráfica a través del Test de Figura humana en niños y con un análisis riguroso, se cuenta con un elemento invalorable para evaluaciones psicológicas/psiquiátricas, específicamente en la determinación del abuso sexual infantil. Esto posibilita disminuir la re victimización en la población afectada si ésta se encuentra en Servicios de Salud Mental y los/as profesionales son convocados desde la Justicia o en la investigación clínica para confirmación de ASI. (12, 14).

Se torna imperioso continuar con estudios que permitan ampliar y mejorar la calidad y precisión de los diagnósticos de ASI en niños, niñas y adolescentes según refieren investigaciones previas $(14,15)$. Porque la población infanto juvenil presenta dificultades para poner en palabras sus sentimientos y expresar sus emociones por lo traumático del hecho vivido, siendo entonces sus expresiones gráficas una alternativa fundamental y privilegiada para acceder a su mundo interno. $(13,14)$.

Como futuras líneas de investigación se necesita la reproducción de este tipo de estudios con mayor tamaño de la muestra en ambos grupos y en población general. 


\section{REFERENCIAS BIBLIOGRAFICAS}

1. UNICEF. Base Educativa y Comunitaria de Apoyo BECA-UNICEF. /Internet/ AsunciónParaguay: UNICEF; 2014. /citado 11 de junio 2013/. Disponible en PDF: http://www.unicef.org/paraguay/spanish/ py_resources_Estudio_Maltrato.pdf

2. Organización Internacional del TrabajoDirección General de Estadísticas Encuestas y Censos. Programa Internacional para la Erradicación del Trabajo Infantil (IPEC). /Internet/. Asunción-Paraguay: DGEEC. /citado 15 de Agosto de 2015/. Disponible en PDF: http://www.dgeec.gov.py/Mag. \%20y\%20 ctca\%20del\%20trabajo\%20infantil\%20y \%20adolescent\%20en\%20el\%20py.pdf

3. Denuncia sobre violencia contra niños aumentaron desde mayo. Diario abc color. 24 de junio de 2015. Disponible en: http://www.abc.com.py/edicionimpresa/locales/denuncias-sobreviolencia-contra-ninos-aumentarondesde-mayo-1380437.html

4. Fondo de las Naciones Unidas para la Infancia. Conceptos vinculados a la explotación sexual infantil definiciones. /Internet/ Buenos Aires-Argentina:UNICEF /citado 15 de Agosto de 2015/. Disponible en http://www.unicef.org/argentina/spanish/ definiciones.pdf

5. Arrom C, Ruoti M, Orué E, Arrom CM. El castigo y sus diferentes formas en la crianza de niños, niñas y adolescentes en 1049 familias de Alto Paraná. Revista Paraguaya de Psiquiatría. 2015;3(1):2632.

6. Arrom C, Fresco MP, Arrom CM, Arce A, Arrom MA, Romero $M$, et al. Violencia intrafamiliar. Revista Paraguaya de Psiquiatría. 2015;3(1):38-41.

7. BASE-Plan-MEC. Estudio exploratorio sobre Maltrato Escolar en Paraguay. /Internet/Asunción-Paraguay. /citado 15 de Agosto de 2015/. Asunción: MEC; 2014. Disponible en PDF: http://www.mec.gov.py/cms_v2/adjuntos /10396

8. Boletín de información y análisis SERPAJ. Infancias destruidas. /Internet/ Editoria L. Año 4 - No 14 | Mayo - 2015. Disponible en PDF:

http://serpajamericalatina.org/nuevaweb/ wp-content/uploads/2015/06/BOLETINDE-INFORMACI\%C3\%93N-YAN\%C3\%81LISIS-SERPAJ-NRO.-142015.pdf
9. Última Hora.com. En cinco años se triplicaron denuncias por abuso sexual en niños. /Internet/. Última Hora. /citado 15 de Agosto de 2015/. Disponible en PDF: http://www.ultimahora.com/en-cincoanos-se-triplicaron-denuncias-abusosexual-ninos-n623405.html

10. Pereda Beltrán N, Gallardo-Pujol N, Jiménez Padilla R. Trastornos de personalidad en víctimas de abuso sexual infantil. Revisión. Actas españolas de psiquiatría, ISSN 1139-9287, 2011; 39 (2): 131-39 Disponible en: http://dialnet.unirioja.es/servlet/articulo? codigo $=3424661$

11. López Y, Gigato B, Álvarez I. Consecuencias Psicológicas del Abuso Sexual Infantil. Eureka /online/. 2012 /citado 2015-08-03/; 9(1):58-68. Disponible en: http://pepsic.bvsalud.org/scielo.php?scrip $\mathrm{t}=\mathrm{sci}$ arttext\&pid=S2220$90262012000100007 \& \mid \mathrm{ng}=$ pt\&nrm $=$ iso $>$. ISSN 2220-9026.

12. Boscato A, Ortalli I, Sobrero D. Dibujos que Hablan: Indicadores de Abuso Sexual Infantil en Gráficos. 2da edición. QuilmesBuenos Aires. Tiempo Sur Ediciones; 2010.

13. Rodríguez López Y, Aguiar B, Álvarez I. Consecuencias psicológicas del abuso sexual infantil. Centro de Documentación. Investigación y Difusión de la Carrera de Psicología, Universidad Católica Ntra. Sra. De la Asunción. Eureka. 2012;9(1):58-68.

14. Opazo Baeza V, Rivera Toledo J.

Indicadores gráficos de la prueba del Dibujo de la Figura Humana en adolescentes hombres y mujeres de 12 a 16 años víctimas de agresiones sexuales. Revista de Psicología /internet/. 2010. Fecha de acceso: 03 ago. 2015 doi: $10.5354 / 0719-$ 0581.2010.17099;19(1):80-107. ISSN 0719-0581. Disponible en: http://www.revistas.uchile.cl/index.php/R DP/article/view/17099/17829.

15. Organización Mundial de la Salud-Oficina Regional para las Américas-Organización Panamericana de la Salud. Comprender y abordar la violencia contra las mujeres. Violencia infligida por la pareja /Internet/. Washington D.C., EEUU: OPS-OMS; 2013. /citado 2 abril 2014/. Disponible en: http://apps.who.int/iris/bitstream/10665/ 98816/1/WHO_RHR_12.36_spa.pdf?ua =1 